Review Article

\title{
Psychology of pandemics and fear of epidemic
}

\author{
M. Naveed Uddin ${ }^{1 *}$, Mohsin Uddin² \\ School of Social Sciences ${ }^{1}$ Bharathiar University, ${ }^{2}$ Indira Gandhi National Open University, India \\ Received: 10 March 2021 \\ Revised: 15 April 2021 \\ Accepted: 19 April 2021 \\ *Correspondence: \\ Dr. M. Naveed Uddin, \\ E-mail: mdnaveid@gmail.com \\ Copyright: (C) the author(s), publisher and licensee Medip Academy. This is an open-access article distributed under \\ the terms of the Creative Commons Attribution Non-Commercial License, which permits unrestricted non-commercial \\ use, distribution, and reproduction in any medium, provided the original work is properly cited.
}

\begin{abstract}
Infectious outbreaks have stirred the psychological health and mental wellbeing of individuals on a global basis. It is evident from the 1918 influenza in addition to the current COVID-19. Psychological factors are significant for comprehension and overseeing cultural issues related with pandemics, like the spreading of extreme dread, anxiety, and xenophobia that happen when individuals are compromised with infection. The research aims to identify how the COVID-19 pandemic has affected individual's psychological wellbeing and how the psychodynamic approach can resolve such an issue. Developing a sense of detachment from oneself and the growing fear of being infected significantly affects an individual psychological wellbeing. This analysis concentrates predominantly on applying the psychodynamic approach to ascertain individual's psychological threats during the COVID-19 crisis. This research focuses on revealing the variations generated from the psyche of individuals due to the COVID-19. It also aims to highlight the impact that COVID-19 have created on the mental health of individual beings. Moreover, the pandemic consequence on the individual's overall lifestyle has discussed in the study.
\end{abstract}

Keywords: Psychodynamic approach, Mental illness, Psychological implications, Epidemic outbreaks, Risk factors of COVID-19

\section{INTRODUCTION}

Pandemics have been affecting humans' psychology and harming society, and a sense of fear is also taking place over people's psychology. An active spread of Coronavirus affects the lifestyle and has changed the methods of working in differing sectors. This study focuses on the risk factors for mental illnesses created due to pandemic and epidemic outbreaks. It also aims to focus on the psychological implications affecting the well-being of the people during the pandemic. It has also sought to identify how mental health prevention can be helpful by applying a psychodynamic approach.

\section{BACKGROUND OF THE STUDY}

The psychological implications attached to a pandemic indicate the inherent fear among humans regarding uncertainties. Individuals are an uncertain threat when they are made aware of an invisible contagion that is fatal and difficult in protecting themselves against. ${ }^{1}$ Researchers have observed that different threats are responsible for pushing other psychological buttons. For instance, novel threats have known to provoke anxiety among individuals due to their fatality and unfamiliarity. Psychologists' role in assisting government and healthcare leaders in communicating with the public during a pandemic is undeniable. This research focuses on analysing the psychological implications associated with the on-going pandemic due to COVID-19.

Diseases that are fatal and familiar are less likely to instigate psychological reactions of fear and anxiety among individuals than alien threats. Researchers have observed that influenza is far more probable to kill more people than any other infectious disease and is 
responsible for killing thousands of people globally each year. ${ }^{2}$ However, novel threats like Ebola and COVID-19 lead to a sense of immediate personal risk urging individuals to take preventive measures. Developing a sense of detachment from oneself and the growing fear of being infected significantly affects individual psychological well-being. This study focuses predominantly on applying the psychodynamic approach to ascertain individual psychological threats during the COVID-19 crisis and identify the psychological issues faced due to the outbreak.

Managing the psychological implications leading to disruption of social behaviour due to increased fear and anxiety affects individual's mental wellbeing. It also indicates that psychological factors play a crucial role in managing maladaptive behaviour caused due to situational uncertainty because of a pandemic. ${ }^{3}$ The study plays an integral role in understanding the impact of COVID-19 on people's psychological wellbeing and its approach to solving public health bodies intrusion.

- To comprehend the status of the psychological imbalance and fear factor among humans due to the arrival of COVID-19.

- To analyse the impact of negative psychology and involvement of fear in individuals.

- To investigate individuals' challenges due to the effect of psychological imbalances and a sense of fear in humans due to complete lockdown in homes.

- To provide recommendations in mitigating the psychological issues and fears among the human to provide wellbeing in society.

\section{PANDEMIC AND EPIDEMIC OUTBREAKS ARE RISK FACTORS FOR MENTAL ILLNESSES}

The psychological factors that cause a direct reaction among individuals in the face of a pandemic when they feel threatened include maladaptive behaviours, defence, and emotional reactions. ${ }^{4}$ It is thereby evident that the larger the outbreak, the larger is its impact on the overall wellbeing of individuals.

The perception of risk during the outbreak of an infectious disease is prominent in such an instance that identifies and manages the psychological implications of a pandemic crucial for developing effective public healthcare systems. Public stigma has been identified to have a detrimental impact on how individuals perceive an outbreak due to their unconscious bias. ${ }^{1}$ For instance, the Ebola outbreak shed light on the mass fear instilled among people living in the US. ${ }^{5}$ Have observed that all healthcare workers and individuals in direct contact with Ebola patients were mandated 21 days of quarantine. ${ }^{5}$ The psychological implications of managing a stressful situation caused due to the pandemic further make it challenging for managing mental illnesses effectively. It is an inherent risk factor for increasing the perception of mental health, making it difficult for them to cope, including depression and anxiety.

In the acute phase during the onset of a pandemic, it is understandable that fear is instilled within individuals. This fear and concern can be amplified by pre-existing anxiety and depressive disorders. Personal reactions differ during an outbreak. ${ }^{4}$ However, the increased thought processes of being inflicted by the disease further increase the likelihood of social and behavioural modification.

\section{PSYCHOLOGICAL IMPLICATIONS AFFECTING THE WELLBEING OF INDIVIDUALS DURING A PANDEMIC}

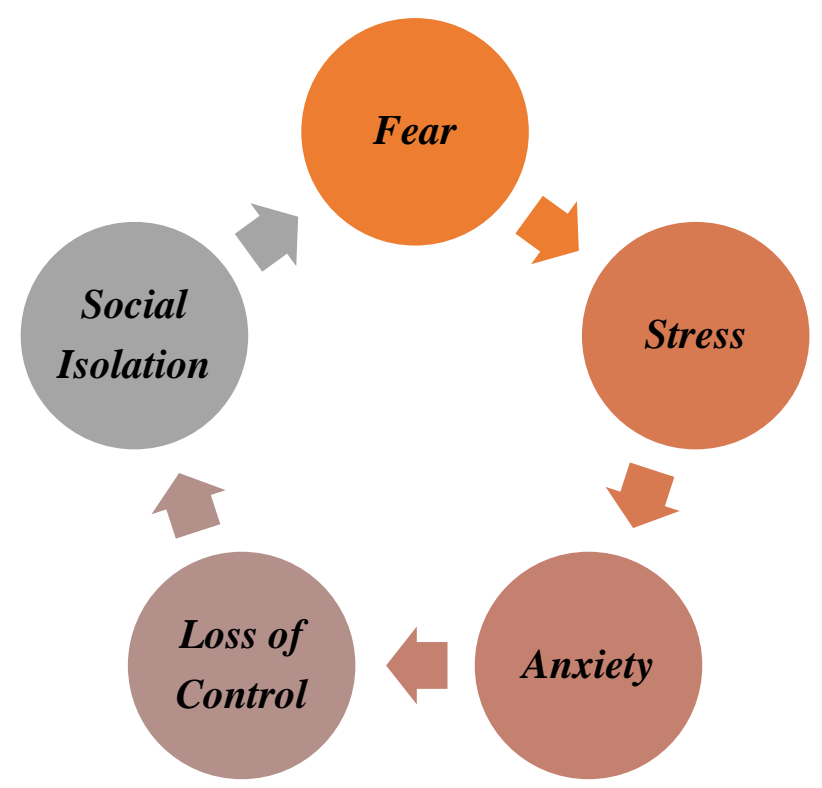

Figure 1: Psychological implications. ${ }^{6}$

Determining the key factors and their impact on an individual's mental wellbeing while managing their lives during a pandemic is crucial for developing appropriate healthcare interventions. Similarities have been found between the existing reactions and psychological implications of epidemics experiences along with the ongoing COVID-19 by researchers. The novel coronavirus indicates an increase in widespread fear, xenophobia, and panic, and stress. It has led to a significant rise in sensitivity towards social risks, decreased positive emotions, and a sense of satisfaction. A rise in fear of being infected has further affected the mental wellbeing of society. A sense of loss of control over oneself and one's surroundings makes individuals vulnerable and increases their distress. ${ }^{6}$ The key psychological implications have been discussed below.

\section{Fear}

Previous epidemics led to widespread fear and loneliness induced among individuals during the onset of the epidemic. The pandemic of COVID-19 generated 
widespread fear among people worldwide, and uncertainty of the situation led to social isolation to prevent the disease's spread. It has eventually affected individuals' emotional and mental well-being and instilled fear among them as the number of deaths continued to rise. The number of people whose mental health is affected tends to last longer than the infection's total number during epidemics. It has been identified that misplaced reactions to a threatening situation trigger a sense of excessive fear among individuals. ${ }^{7}$ The thing indicated the variation in reactions causing stigmatization of the disease to those affected by the situation.

\section{Stress and anxiety}

Novel threats have, as observed by researchers, provoke a sense of anxiety and stress among individuals. The COVID-19 is a stressful event making it crucial for public health bodies to ascertain the process of managing fear, stress, and anxiety among individuals. ${ }^{8}$ On a global basis, pandemic causes anxiety and distress among people. In the areas of war zones and communities affected by violence, people are already facing psychological stress. Anxiety, is noted as a recurrent psychological implication caused during pandemics.

Similarly, during the COVID-19 outbreak, a steep rise in anxiety among individuals has been evident worldwide. The COVID-19 pandemic has led to a direct impact on individuals' mental well-being across the globe, triggering a rise in anxiety and stress due to the unknown contagion and its widespread wreckage of daily lives. Mental Health support is a vital response to adapting to these kinds of psychological issues. In addition to that, support through the provision of psychological and technical help by front-line workers and staff workers is an immediate response to this kind of situation.

\section{Loss of control}

Loss of control over the ability to overcome the pandemic is a threat observed since the influence outbreak in 1918. The implementation of a nationwide lockdown due to the pandemic leads to a disruption of the public's daily life, which directly impacts the economy and livelihood of the people. The loss of control over their lives due to the temporary closure of economic and social activities leads to anxiety, fear, and depression. Post-lockdown paranoia and fear have been increasing as common triggers affecting the individual's mental well-being due to the overarching feelings of loss. In addition to this, loss of jobs and income has created extended effects on the individuals' psyche. A steep rise in mental health issues has noted, leading to delusional thinking that has activated an upsurge in anxiety among individuals during the H1N1 outbreak. ${ }^{9}$ Intensification of fear among individuals due to a loss of control over one's ability to manage the situation leads to further escalating mental health issues, including depression and anxiety.

\section{Importance of understanding psychological implications during a pandemic}

Understanding the psychological implications of an ongoing pandemic is essential to counter situational anxiety, fear, and stress. Past epidemics and pandemics have indicated individuals' ability to discriminate and stigmatize individuals afflicted by the disease. ${ }^{10}$ The key issues that arise due to psychological factors during an outbreak involve fear, stigmatization, along xenophobia. Xenophobia towards Chinese people due to COVID-19 has been indicated to have accelerated globally. ${ }^{10}$ Researchers have noticed xenophobia related to the novel virus and fear of infections, showing a similar psychology pattern among individuals and their responses similar to past epidemics. Management of excessive fear and anxiety during a pandemic is crucial for maintaining social order. ${ }^{11}$ The key similarities between the perception of mental illnesses and that of infectious diseases lead to public stigmatization.

\section{MANAGING THE PSYCHOLOGICAL IMPLICATIONS RELATED TO PANDEMIC AND EPIDEMICS AMONG INDIVIDUALS IS CRUCIAL FOR PREVENTING SOCIAL DISRUPTION}

Effective management of the psychological implications associated with the outbreak of a pandemic or an epidemic is essential. It has been observed that over 21 million tweets regarding Ebola were reported in the US alone. ${ }^{5}$ The UK's roots and US-led to an increase in a misunderstanding of the diseases, causing increased panic and fear among the common masses in the two countries. The media must ensure that the psychological implications associated with such claims are addressed use sensible communication and informative messages. ${ }^{12}$ Similarly, during the H1N1 influenza outbreak, honest and accurate reporting of the situation by the Swedish government and media houses led to realistic management of expectations of the public and their fear. ${ }^{5}$ By ensuring the health risks and uncertainty of the situation, the Swedish government heightened the anticipation of the epidemic and shed light on the measures to protect themselves.

On the other hand, Australian media outlets accurately reported the pandemic but focused on its public agency's missteps more. This led to an increased sense of loss of control, fear, and anxiety among people living in Australia. Researchers identified that individuals who could not tolerate uncertainty experienced more stress during the pandemic. ${ }^{12}$ These individuals were least likely to believe that the situation would improve and that they could protect themselves. It is thereby evident that accurately framing informative messages by focusing on acknowledging psychological implications is essential for managing the same during an outbreak. 


\section{APPLICATION OF INTERVENTION MODELS AND THEORIES FOR MANAGING THE PSYCHOLOGICAL IMPLICATIONS CAUSED DUE TO THE PANDEMIC}

\section{Psychodynamic approach}

The application of Freud's psychodynamic approach to the present pandemic sheds light on the unconscious mind and defence mechanism undertaken by individuals to manage their fears. A recent study noted that the psychodynamic approach indicates that unconscious feelings of anxiety and uncertainty have an undeviating impact on people's behaviour and their reactions to present circumstances. ${ }^{2}$ Although these disturbing thoughts and emotions happen in the person's unconscious mind, they still can influence one's behaviour. This approach would be an effective development of a healthcare intervention dedicated to understanding underlying psychological implications caused due to the COVID-19 and manage the same for psychological growth and wellbeing.

\section{Prospect theory of decision-making}

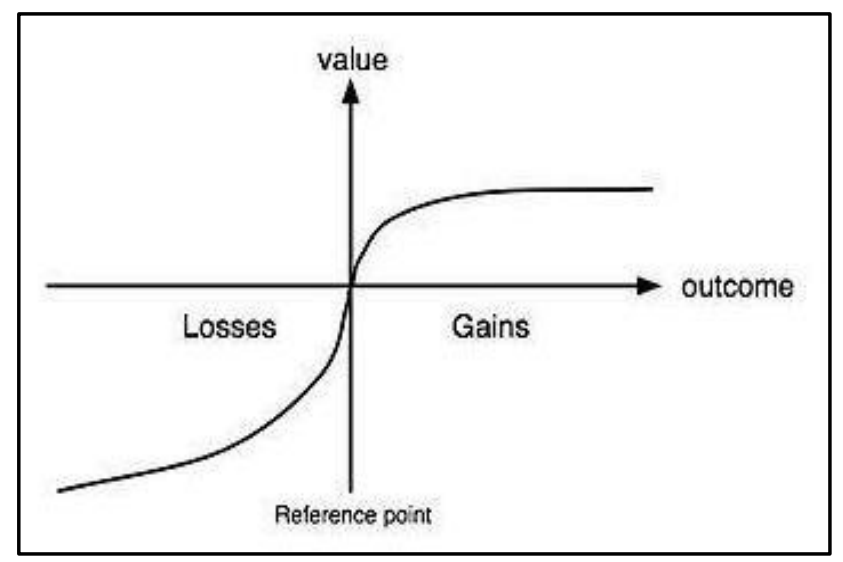

Figure 2: Prospect theory of decision making.

Application of the "prospect theory of decision-making" asserts that individual decisions vary in a given situation. The projection of this graphical picture in the prospect theory of decision making tends to provide features for analysing the losing ability of psychological imbalances, which fails to determine the value. Apart from this, better analysis of fear and psychological imbalances can gain by outreaching the tests' values and outcomes. These decisions depend on the expected loss or gain, and tits ascertained magnitude by an individual. They have identified that the prospect theory focuses on taking the increased extent of risks while the improvements may make an individual lesser prone to risk-taking. ${ }^{13}$ It is applicable for managing high-risk situations like the ongoing pandemic. This theory's application is adequate for navigating the present situation and developing proper intervention methods that increase the likelihood of gains through increased risk-taking.

\section{APPLICATION OF PSYCHODYNAMIC APPROACH IS EFFECTIVE FOR MANAGING THE PSYCHOLOGICAL IMPLICATION CAUSED DUE TO COVID-19}

The COVID-19 has had a direct impact on the psychology and behavioural systems followed by individuals. The psychodynamic approach is essential for identifying and managing individuals and group mental health problems. ${ }^{2}$ The WHO has recommended practicing physical and social distancing among individuals to manage the risk associated with COVID-19. On the other hand, it has been found that people with complex attitudes and psychological imbalances consist of higher chances of being the patient of COVID-19. The presence of this Coronavirus within an individual leads to generate fear, and humans' psychology gets disturbed. Researchers asserted that the application of psychodynamic perspectives enables the public healthcare system to understand problematic behaviour and manage stressors faced by individuals. ${ }^{2}$ It emphasizes the interactions of an individual and the impact that their defence mechanisms have on their behaviour. For instance, "disavowal" is a defence mechanism that individuals use to protect themselves from a situation they do not have control to cope with. Since it is difficult to cope with such behaviour, it leads to a turbulent psychological atmosphere caused by the COVID-19, causing displacement of anger and frustration. It has been observed that the projection of internet sources like online psychological therapy sessions aims to analyse the issues and investigates the fear factor in humans and accordingly provides steps to maintain the patients. ${ }^{14}$ Application of psychodynamics approach for developing public health care policies and providing care is essential for ensuring that apart from one's physical health, their mental wellbeing needs are also catered to. Utilization of the same would help empower the public healthcare system to ensure that general mental wellbeing and psychological implications due to the COVID-19 outbreak are addressed properly. ${ }^{14}$ To manage the psychological consequences of the COVID-19, the following recommendations can be applied by the public healthcare system and policymakers globally.

\section{Inclusion of a psychodynamic approach for developing an effective behavioural modification system}

By including the psychodynamic approach towards developing effective public healthcare response systems to mental health emergencies, the public healthcare system would significantly be able to cope with the increase in anxiety and depression among individuals. Addressing individuals' defence mechanisms as a reaction due to a crisis is essential. ${ }^{14}$ By systematically acknowledging and addressing the underlying triggers for behaviour leading to noncompliance with WHO's preventive measures, Freud's psychodynamic perspective can also be incorporated. 


\section{Access to psychological therapy sessions online}

The evaluation of online psychological therapy sessions improves mental health and resolves the fear factor that arrived because of COVID-19. The projection of online psychology therapy sessions would help in counselling, due to which psychologists able to comprehend the stress factor in humans. Therefore, the evaluation of online psychology therapy sessions would help analyse the stress and fear factor and implement strategic changes in individual's psychology.

\section{Development of accurate risk communication messages}

The development of accurate risk communication to address the psychological fear and anxiety caused due to the COVID-19 would be incredibly effective for ensuring that individuals are aware of the risks. It would further increase the likelihood of compliance with government regulations and policies. By providing accurate information on socially disruptive behaviours of public stigma and xenophobia, the media and healthcare bodies can work collaboratively to ensure that the pandemic is managed. This would reinstate the sense of hope among individuals and help them manage their fear of uncertainty in a better manner.

\section{CONCLUSION}

The importance of identifying and managing psychological wellbeing of individuals is unstable during the pandemic. The uncertainty and fear of being inflicted by the disease is a significant stressor that causes individuals to feel panicked and triggers their anxiety. Identifying psychological implications caused due to the pandemic is the primary step of developing a practical approach for addressing the same. These include social problems in the form of public stigma, xenophobia along with excessive fear and anxiety. It also ensures that positive behavioural modifications can be instilled among individuals to cope with the stressful and turbulent environment resulting from the COVID-19 outbreak.

Funding: No funding sources Conflict of interest: None declared

Ethical approval: Not required

\section{REFERENCES}

1. Khan S, Huremović D. Psychology of the Pandemic. In Psychiatry of Pandemics. Springer, Cham. 2020;37-44.

2. Marčinko D, Jakovljević M, Jakšić N, Bjedov S, Drakulić A. The importance of psychodynamic approach during COVID-19 pandemic. Psychiatria Danubina. 2020;32(1):15-21.
3. Taylor S. EAP and COVID-19: The Psychology of Pandemics. 2020, Available at: https://archive.hshsl.umaryland.edu/handle/10713/12 920. Accessed on 1 Jan, 2021

4. Morganstein JC, Fullerton CS, Ursano RJ, Donato D, Holloway HC. Pandemics: Health care emergencies. Textbook of Disaster Psychiatry. $2^{\text {nd }}$ ed, Cambridge University Pr. 2017;270-83.

5. Apa.org. An epidemic of fear. 2015. Available at: https://www.apa.org/monitor/2015/03/fear. Accessed on 19 June 2020.

6. Pfefferbaum B, North CS. Mental health and the Covid-19 pandemic. N Eng J Med. 2020;383:510-12.

7. Holmes EA, O'Connor RC, Perry VH, Tracey I, Wessely S, Arseneault L et al. Multidisciplinary research priorities for the COVID-19 pandemic: a call for action for mental health science. Lancet Psychiatry. 2020;7(6):547-60.

8. Mesa VC, Franco OH, Gómez RC, Abel T. COVID19: The forgotten priorities of the pandemic. Maturities. 2020;136:38.

9. Imai $H$. Trust is a key factor in the willingness of health professionals to work during the COVID-19 outbreak: Experience from the $\mathrm{H} 1 \mathrm{~N} 1$ pandemic in Japan 2009. Psychiatry and Clinical Neurosciences. 2020;7-4(5):32930.

10. Schimmenti A, Billieux J, Starcevic V. The four horsemen of fear: An integrated model of understanding fear experiences during the COVID19 pandemic. Clin Neuropsychiatry. 2020;17(2):415.

11. Arafat SY, Kar SK, Marthoenis M, Sharma P, Apu EH, Kabir R. Psychological underpinning of panic buying during pandemic (COVID-19). Psychiatry Research. 2020;289:113061.

12. Taha S, Matheson $\mathrm{K}$, Cronin $\mathrm{T}$, Anisman $\mathrm{H}$. Intolerance of uncertainty, appraisals, coping, and anxiety: The case of the $2009 \mathrm{H} 1 \mathrm{~N} 1$ pandemic. Bri j health psycho. 2014;19(3):592-605.

13. Hameleers M. Prospect Theory in Times of a Pandemic: The Effects of Gain versus Loss Framing on Policy Preferences and Emotional Responses During the 2020 Coronavirus Outbreak. 2020.

14. Békés V, Aafjes-van Doorn K. Psychotherapists' attitudes toward online therapy during the COVID-19 pandemic. J Psychotherapy Integration. 2020;30(2):238.

Cite this article as: Uddin MN, Uddin M.

Psychology of pandemics and fear of epidemic. Int J Community Med Public Health 2021;8:2592-6. 